\title{
Does water enhance Mg intercalation in oxides? The case of a tunnel framework
}

Mario Lopez, ${ }^{a, b}$ Hyun Deog Yoo ${ }^{a, b, c}$ Linhua Hu ${ }^{a, b}$ Justin L. Andrews, ${ }^{d}$ Sarbajit Banerjee ${ }^{d}$ and Jordi Cabana ${ }^{\mathrm{a}, \mathrm{b}, *}$

${ }^{a}$ Department of Chemistry, University of Illinois at Chicago, Chicago, Illinois 60607, USA

b Joint Center for Energy Storage Research, Argonne National Laboratory, Lemont, Illinois 60439, USA

c Department of Chemistry and Chemical Institute for Functional Materials, Pusan National University, Pusan 46241, Korea

${ }^{\mathrm{d}}$ Department of Chemistry, Texas A\&M University, College Station, TX 77843, USA

*Corresponding author, E-mail: jcabana@uic.edu

\section{Supporting Information}




\section{Methods}

Preparation of $\zeta-\mathrm{V}_{2} \mathrm{O}_{\mathbf{5}}$ electrodes: $\zeta-\mathrm{V}_{2} \mathrm{O}_{5}$ was synthesized from $A g_{1 / 3} \mathrm{~V}_{2} \mathrm{O}_{5}$ using the procedure demonstrated by Andrews et al. ${ }^{1}$ Briefly, the precursor material, $\beta-\mathrm{Ag}_{x} \mathrm{~V}_{2} \mathrm{O}_{5}$ (where $x \sim 0.33$ ) was synthesized by a hydrothermal reaction between $\mathrm{AgCOOCH}_{3}$ (Sigma Aldrich) and $\mathrm{V}_{2} \mathrm{O}_{5}$ (Sigma Aldrich). The precursor material was treated hydrothermally in an acidic solution (15.8 $\mathrm{ml}$ of $0.59 \mathrm{M} \mathrm{HCL}$ (aq.)) at $210^{\circ} \mathrm{C}$ for $24 \mathrm{~h}$, according to the following, previously reported, reaction: ${ }^{2}$

$$
\beta-\mathrm{Ag}_{0.33} \mathrm{~V}_{2} \mathrm{O}_{5}(\mathrm{~s})+0.28 \mathrm{HCl} \text { (aq.) }+0.145 \mathrm{O}_{2}=\zeta-\left(\mathrm{Ag}_{0.05}\right) \mathrm{V}_{2} \mathrm{O}_{5}(\mathrm{~s})+0.28 \mathrm{AgCl}(\mathrm{s})+0.14 \mathrm{H}_{2} \mathrm{O}
$$

Note the role of dissolved oxygen as the oxidizing agent. The as-prepared material had a small amount of residual $\mathrm{Ag}$ in the structure $\left(\sim 0.05 \mathrm{~mol}\right.$ per mol $\left.\mathrm{V}_{2} \mathrm{O}_{5}\right),{ }^{1}$ which explains its slightly lower oxidation state of $\mathrm{V}$ compared to $\alpha-\mathrm{V}_{2} \mathrm{O}_{5}$ (Figure 2a). However, previous HAXPES, XAS, and XES measurements have corroborated the primarily +5 oxidation state of $\zeta-\mathrm{V}_{2} \mathrm{O}_{5}$ nanowires upon topochemical deintercalation of Ag-ions. $\alpha-\mathrm{V}_{2} \mathrm{O}_{5}$ is nominally expected to be exclusively $\mathrm{V}^{5+}$; however, previous studies have shown that $\alpha-\mathrm{V}_{2} \mathrm{O}_{5}$ still has some $\mathrm{V}^{4+}$ character (likely due to the formation of oxygen vacancies). ${ }^{3}$ The slightly reduced state of $\zeta-\mathrm{V}_{2} \mathrm{O}_{5}$ relative to $\alpha-\mathrm{V}_{2} \mathrm{O}_{5}$ has been noted in previous work ${ }^{3}$ and is wholly consistent with partial reduction of the material due to residual silver that is not completely removed during the topochemical extraction process. Note that in recent work orange-colored single crystals of $\zeta-\mathrm{V}_{2} \mathrm{O}_{5}$ have been isolated from topochemical deintercalation of $\mathrm{Cu}^{+}$from $\beta^{\prime}-\mathrm{Cu}_{x} \mathrm{~V}_{2} \mathrm{O}_{5}$ and $\mathrm{Li}^{+}$from $\beta^{\prime}-\mathrm{Li}_{x} \mathrm{~V}_{2} \mathrm{O}_{5}$ in a dry aprotic solvent, $\mathrm{CH}_{3} \mathrm{CN}$ further ruling out substantial proton occupancy within the tunnels. ${ }^{4}$

Preparation of Ionic Liquid Electrolyte with $\mathbf{M g}^{2+}$ Ions: In argon-filled glovebox, magnesium bis(trifluoromethylsulfonyl)imide (MgTFSI ${ }_{2}$, Solvionic) salt was mixed with $N$-methyl- $N$ butylpyrrolidinium bis(trifluoromethylsulfonyl)imide (PY14TFSI, Solvionic) to make a solution of $0.5 \mathrm{M} \mathrm{MgTFSI}_{2}$. Once the liquid mixture was thoroughly mixed, the electrolyte was kept under vacuum at $110{ }^{\circ} \mathrm{C}$ for 50 hours to eliminate $\mathrm{H}_{2} \mathrm{O}$ introduced with the starting reagents. This electrolyte mixture is labeled as "Dry IL." Preparation of "Wet IL" electrolyte only involved the same mixtures, but skipped the drying step. Karl-Fischer Titration was performed on both electrolyte mixtures. 
The relative water content was cross-checked with Fourier-transform infrared (FTIR) spectroscopy, using a Nicolet iS5 (Thermo Scientific) spectrometer (Figure S1). The main difference between the samples from dry and wet electrolytes was the presence of two peaks in Wet IL at 1700 and $3500 \mathrm{~cm}^{-1}$; among them, the latter peak was significantly broader. These signals are easily assigned to the bending and stretching modes of $\mathrm{H}_{2} \mathrm{O}$, respectively, confirming the difference in moisture content introduced by the drying step. $\mathrm{H}_{2} \mathrm{O}$ was essentially undetectable in Dry IL in consistence with the very low concentration measured by Karl-Fischer titration, a more sensitive method than FTIR.

Electrochemical Measurements: Working electrodes were composed of a 3:1:1 mass ratio that was comprised from $\zeta-\mathrm{V}_{2} \mathrm{O}_{5}$, acetylene black, and 6 wt.\% Polyvinylidene Fluoride (PVDF, Solvay) in 1-methyl-2-pyrrolidinone (NMP, Sigma Aldrich, $>99 \%$ ). First, $\zeta-\mathrm{V}_{2} \mathrm{O}_{5}$ and acetylene black was grinded into fine powder by mortar. Next, a solution of $6 \mathrm{wt} \%$. polyvynilidene difluoride (PVDF) in N-methylpyrrolidone (NMP) was added to the powder mixture and mixed to form a slurry. The black slurry was then quickly applied to coat the stainless-steel mesh. The stainlesssteel mesh substrates had an area of $0.97 \mathrm{~cm}^{2}$. Afterwards, the electrodes were then dried under a heat lamp for 30 minutes. After drying, the electrodes were flattened by applying at least $3000 \mathrm{psi}$ of pressure and then stored in a vacuum oven at $80^{\circ} \mathrm{C}$ overnight.

Two-electrode coin-type cells were used, with the active material as the working electrode, glass fiber as the separator (VWR, grade $691,210 \mu \mathrm{m}, 2 \mathrm{~cm}^{2}$ ), and activated carbon cloth as the counter electrode (ACC-5092-20, Kynol Co.). The cells were constructed in an argon-filled glovebox. The cells were cycled at the rate of $\mathrm{C} / 20$ in a $110^{\circ} \mathrm{C}$ oven with forced convection (Binder Inc.). All electrochemical experiments utilized a multi-channel potentiostat (VMP3, Bio-Logic). By the calibration against ferrocene/ferrocenium couple ( $c a .2 .5 \mathrm{~V} \mathrm{vs} \mathrm{Mg} / \mathrm{Mg}^{2+}$ ), it was found that the open circuit potential of $\mathrm{ACC}$ is $2.2 \mathrm{~V}$ higher than $\mathrm{Mg} / \mathrm{Mg}^{2+}$. After the electrochemical cycling of the two-electrode cells, the genuine potential of the cathode was estimated by considering the potential shift of ACC counter electrode, which is linearly proportional to the state-of-charge $(\mathrm{SoC})$ as a capacitive electrode.

Characterization of Materials/Electrodes: X-ray diffraction (XRD) of electrodes was performed in a custom air-free sample holder, using a Bruker D8 Advance diffractometer with $\mathrm{Cu} \mathrm{Ka}$ radiation $(\lambda=1.5418 \AA$ ); and the patterns were fit using the Pawley method to index the unit cell 
lattice. V $K$-edge X-ray absorption spectroscopy (XAS) measurements were performed by the MRCAT bending magnet in 10-BM beamline of the advanced photon source (APS) at Argonne national laboratory. The XAS spectra were collected in a transmission mode through composite electrode laminates. A $\mathrm{Si}(311)$ water-cooled double-crystal monochromator was used to scan incident X-ray and scanned energies were detuned by 50 percent. The beam profile was collimated to $3 \times 0.75 \mathrm{~mm}$. A V reference foil was measured simultaneously with every sample for energy calibration (V K-edge set to $5645 \mathrm{eV}$ ). Transmission electron microscopy (TEM) was performed using an aberration-corrected microscope (JEOL, Model 3010) operated at $200 \mathrm{kV}$; energydispersive X-ray (EDX) spectra were collected using a high-solid angle Oxford X-MaxN 100TLE silicon drift detector that is attached to the TEM. FTIR spectra of electrodes were measured by Nicolet iS5 (Thermo Scientific) spectrometer in attenuated total reflection (ATR) mode.

\section{Bibliography:}

1. Andrews, J. L.; Mukherjee, A.; Yoo, H. D.; Parija, A.; Marley, P.; Fakra, S.; Prendergast, D.; Cabana, J.; Klie, R. F.; Banerjee, S., Reversible Mg-Ion Insertion in a Metastable One-Dimensional Polymorph of V2O5. Chem 2018, 4, 564-585.

2. $\quad$ Marley, P. M.; Abtew, T. A.; Farley, K. E.; Dennis, R. V.; Horrocks, G. A.; Zhang, P.; Banerjee, S., Emptying and Filling a Tunnel Bronze. Chem. Sci. 2015, 6, 1712-1718.

3. Tolhurst, T. M.; Leedahl, B.; Andrews, J. L.; Marley, P. M.; Banerjee, S.; Moewes, A., Contrasting 1D Tunnel-Structured and 2D Layered Polymorphs of V2O5: Relating Crystal Structure and Bonding to Band Gaps and Electronic Structure. Phys. Chem. Chem. Phys. 2016, 18, 15798-15806.

4. Handy, J. V.; Luo, Y.; Andrews, J.; Bhuvanesh, N.; Banerjee, S., An Atomic View of Cation Diffusion Pathways from Single-Crystal Topochemical Transformations. Angew.Chem. Int. Ed. Early View. 


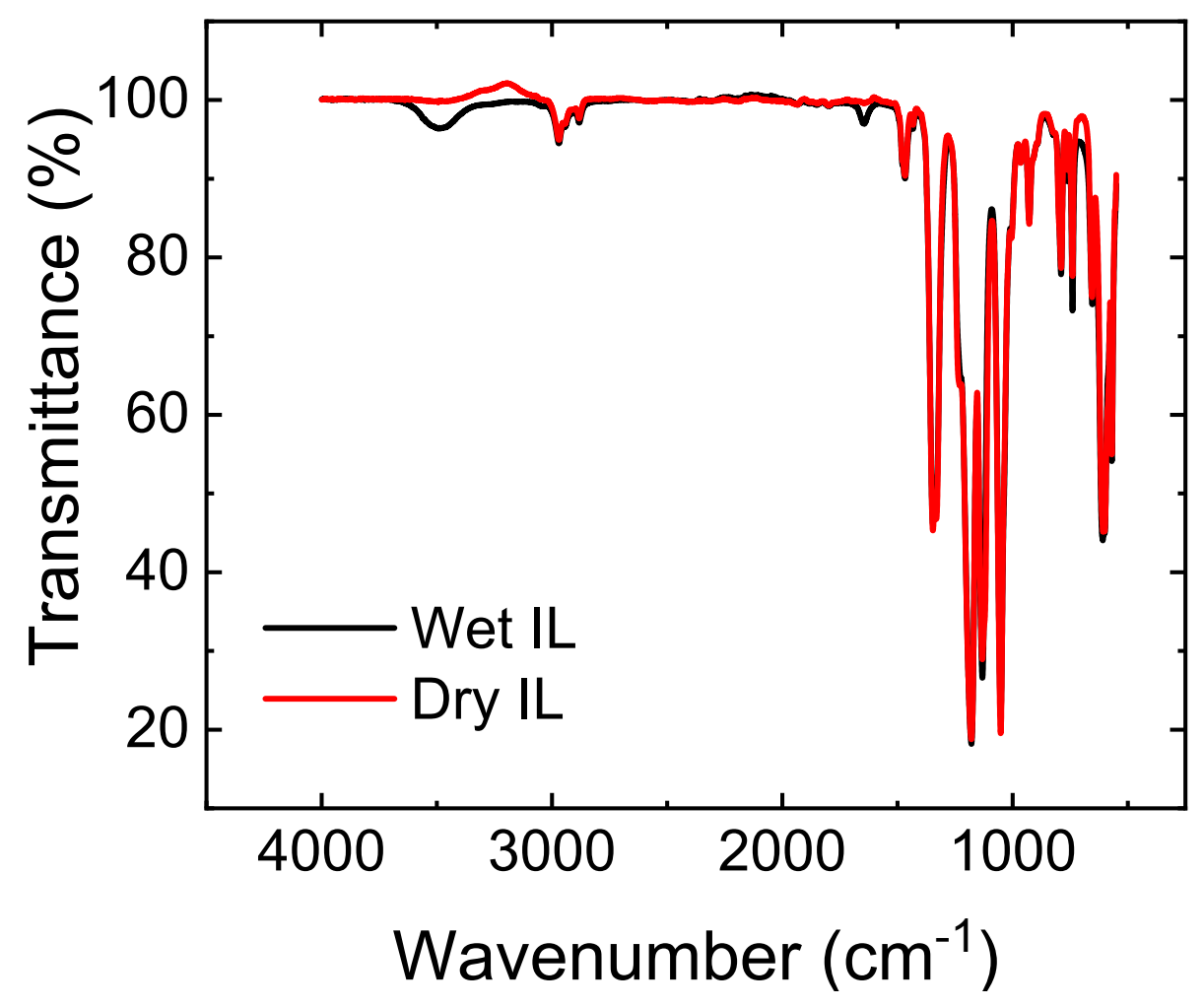

Figure S1. FTIR spectra of Dry IL (red) and Wet IL (black). 

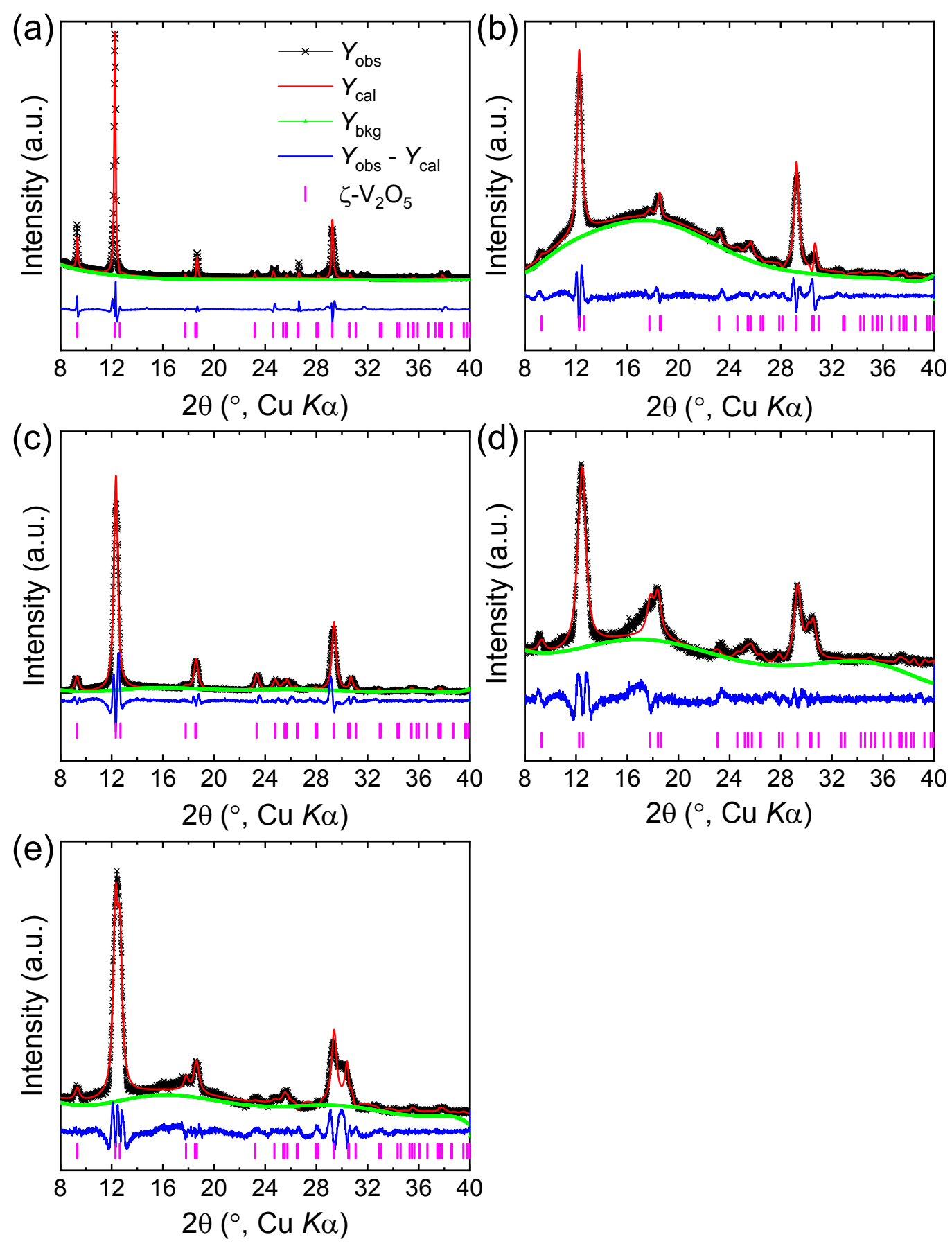

Figure S2. Pawley refinements of XRD patterns of different samples: (a) pristine, (b) reduced and (c) re-oxidized in Dry IL, and (d) reduced and (e) re-oxidized in Wet IL. Black crosses represent the observed intensities; red, green, and blue lines represent the calculated patterns, the background, and the difference between the observed and calculated intensities, respectively. Magenta short vertical bars indicate the position of Bragg reflections of the space group $C 2 / \mathrm{m}$. 


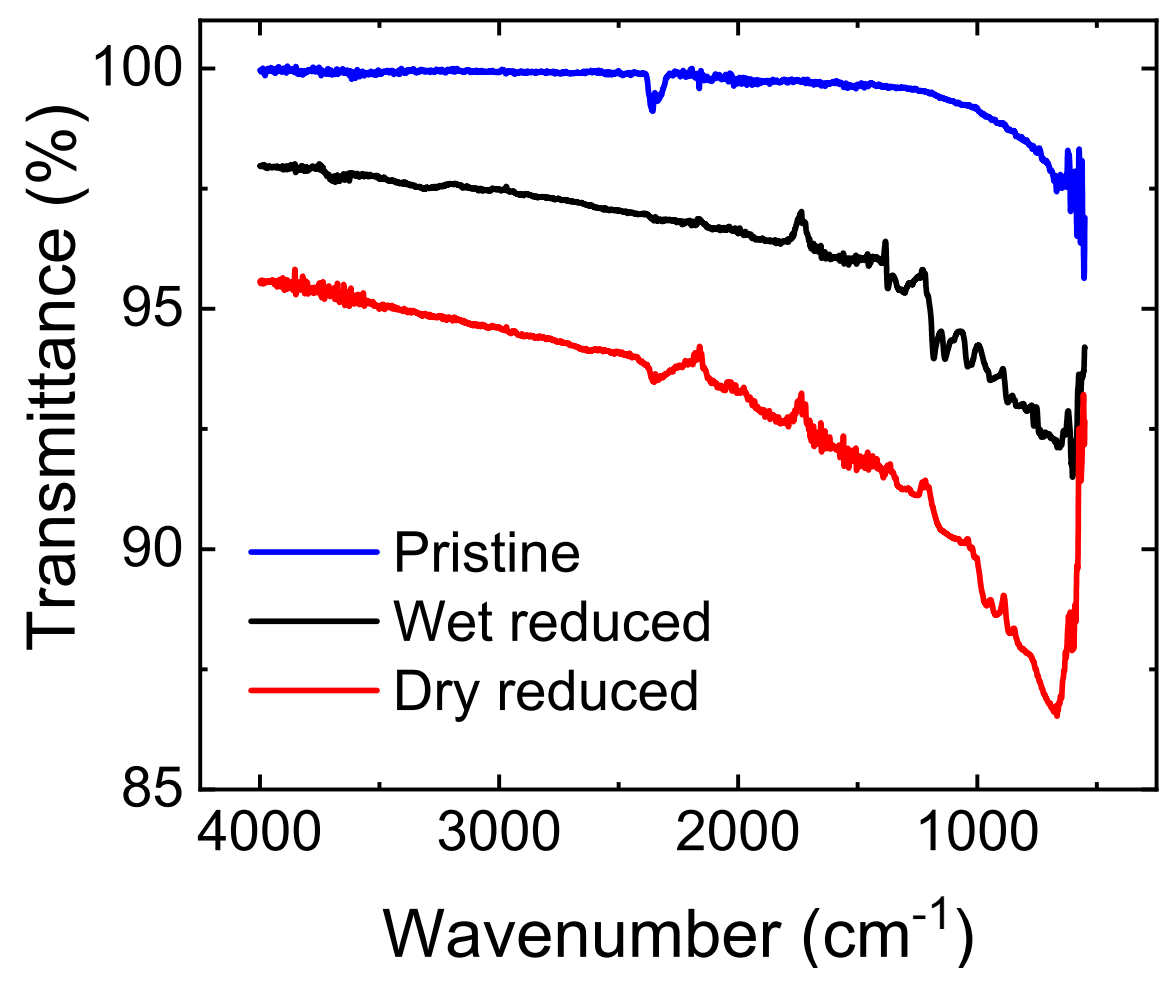

Figure S3. FTIR spectra of $\zeta-\mathrm{V}_{2} \mathrm{O}_{5}$ electrodes that were reduced in Dry IL (red) and Wet IL (black), compared with the pristine state (blue). 
